\begin{tabular}{|c|l|}
\hline Title & Structure and decay pattern of the linear-chain state in C-14 \\
\hline Author(s) & Baba, T.; Kimura, M. \\
\hline Citation & $\begin{array}{l}\text { Physical review C, 94(4), 044303 } \\
\text { https://doi.org/10.1103/PhysRevC.94.044303 }\end{array}$ \\
\hline Issue Date & 2016-10-07 \\
\hline Doc URL & http://hdl.handle.net/2115/63899 \\
\hline Rights & ○2016 A merican Physical Society \\
\hline Type & article \\
\hline File Information & PhysRevC.94.044303.pdf \\
\hline
\end{tabular}

Instructions for use 


\title{
Structure and decay pattern of the linear-chain state in ${ }^{14} \mathrm{C}$
}

\author{
T. Baba and M. Kimura \\ Department of Physics, Hokkaido University, 060-0810 Sapporo, Japan
}

(Received 1 June 2016; published 6 October 2016)

\begin{abstract}
The linear-chain states of ${ }^{14} \mathrm{C}$ are theoretically investigated by using the antisymmetrized molecular dynamics. The calculated excitation energies and the $\alpha$ decay widths of the linear-chain states were compared with the observed data reported by recent experiments. The properties of the positive-parity linear-chain states reasonably agree with the observation, which convinces us of the linear-chain formation in the positive-parity states. On the other hand, in the negative-parity states, it is found that the linear-chain configuration does not correspond to a single eigenstate but is mixed with other configurations in the eigenstates and does not form a single rotational band. As a further evidence of the linear-chain formation, we focus on the $\alpha$-decay pattern. It is shown that the linear-chain states decay to the excited states of daughter nucleus ${ }^{10} \mathrm{Be}$ as well as to the ground state, while other cluster states dominantly decay into the ground state. Hence, we regard that this characteristic decay pattern is a strong signature of linear-chain formation.
\end{abstract}

DOI: 10.1103/PhysRevC.94.044303

\section{INTRODUCTION}

A variety of the $\alpha$ cluster structure is known to exist in light stable nuclei. The most famous example is the Hoyle state (the $0_{2}^{+}$state of ${ }^{12} \mathrm{C}$ ) whose dilute gas-like $3 \alpha$ cluster structure has been studied in detail [1-8] and is well identified today. The linear-chain configuration of $3 \alpha$ clusters, in which $\alpha$ particles are linearly aligned, is another example of famous and exotic cluster structure. It was first suggested by Morinaga [9] to explain the structure of the Hoyle state. However, as mentioned above, it turned out that the Hoyle state does not have the linear-chain configuration but has a dilute gas-like nature. In addition to this, the instability of the linear-chain configuration against the bending motion (deviation from the linear alignment) was pointed out by the antisymmetrized molecular dynamics (AMD) [4] and fermionic molecular dynamics (FMD) calculations [7]. Thus, the formation of a perfectly linear-aligned configuration in ${ }^{12} \mathrm{C}^{*}$ looks negative despite many years of research.

The interest in the linear-chain configuration is reinforced by the physics of unstable nuclei because the valence neutrons may stabilize it by their glue-like role. Such a glue-like role of valence neutron is well known for Be isotopes in which the $2 \alpha$ cluster core is assisted by the valence neutrons occupying the molecular orbits [10-15]. As a natural consequence, we expect that the linear-chain configuration can be realized in neutronrich $\mathrm{C}$ isotopes, and this expectation has been motivating many theoretical and experimental studies [16-28]. Recently, rather promising candidates of linear-chain configuration in ${ }^{14} \mathrm{C}$ were independently reported by two groups [29,30]. Both groups observed the ${ }^{4} \mathrm{He}+{ }^{10} \mathrm{Be}$ resonances above the $\alpha$-threshold energy in both of positive and negative parity. The reported energies of the positive-parity resonances measured from the $\alpha$ threshold are in reasonable agreement with the excitation energies of the linear-chain states predicted by Suhara et al. [24] on the basis of the antisymmetrized molecular dynamics (AMD) calculation. Thus, rather promising evidence of the linear-chain formation has been found.

However, there are still several gaps between theory and experiment which must be resolved to confirm the linear-chain formation in ${ }^{14} \mathrm{C}$. First, when measured from the ground-state energy, theoretically predicted and experimentally observed excitation energies of the positive-parity resonances disagree. This may be because the effective interactions used in the calculation [31] do not reproduce the $\alpha$-threshold energy. Second, the experiments report negative-parity resonances, while the negative-parity linear-chain states were not clearly identified in Ref. [24]. Finally, the experiment of Ref. [29] reported the $\alpha$-decay width of the resonances which is strong evidence of $\alpha$ clustering and must be verified by the theoretical calculations. Thus, further theoretical studies are needed to identify the linear-chain states in ${ }^{14} \mathrm{C}$.

For this purpose, we investigated the linear-chain states in ${ }^{14} \mathrm{C}$. For the sake of quantitative comparison of the excitation energy, we performed AMD calculation employing the Gogny D1S effective interaction [32], which reproduces threshold energies in ${ }^{14} \mathrm{C}$. From the AMD wave function, we estimated the $\alpha$ decay widths of the linear-chain states as well as those of other cluster and noncluster states. It is found that the calculated excitation energies of the positive-parity linear-chain states are in good agreement with the observation, and only the linear-chain states have large $\alpha$ decay widths comparable with the observed data. Hence, we consider that the linear-chain formation in the positive-parity state is rather plausible. On the other hand, in the negative-parity state, the linear-chain configuration does not correspond to a single eigenstate but is mixed with other configurations in the eigenstates. Thus, the linear-chain configuration does not form a single rotational band. As further evidence of the linear-chain formation, we focus on the $\alpha$-decay pattern. It is shown that the linear-chain states decay to the excited states of ${ }^{10} \mathrm{Be}$ as well as to the ${ }^{10} \mathrm{Be}$ ground state, while other cluster states dominantly decay to the ${ }^{10} \mathrm{Be}$ ground state. This characteristic decay pattern is, if it is observed, a strong signature of linear-chain formation.

The contents of this paper are as follows: In the next section, the AMD framework and the method to estimate the $\alpha$-decay width are briefly outlined. In Sec. III, the results of the energy variation and generator coordinate method are presented. In the Sec. IV, the energies and $\alpha$ width of the linear-chain states 
are compared with the observed data. In the last section, we summarize this study.

\section{THEORETICAL FRAMEWORK}

\section{A. Variational calculation and generator coordinate method} reads

The microscopic A-body Hamiltonian used in this study

$$
H=\sum_{i=1}^{A} t_{i}+\sum_{i<j}^{A} v_{i j}^{N}+\sum_{i<j}^{Z} v_{i j}^{C}-t(\text { c.m. })
$$

where the Gogny D1S interaction [32] is used as an effective nucleon-nucleon interaction $v^{N}$. It is shown that the Gogny D1S interaction reasonably describes the one-neutron-, $\alpha$ - and ${ }^{6} \mathrm{He}$-threshold energies within $1 \mathrm{MeV}$ error. The Coulomb interaction $v^{C}$ is approximated by a sum of seven Gaussians. The center-of-mass kinetic energy $t$ (c.m.) is exactly removed.

The intrinsic wave function $\Phi_{\text {int }}$ of AMD is represented by a Slater determinant of single-particle wave packets,

$$
\Phi_{\text {int }}=\mathcal{A}\left\{\varphi_{1}, \varphi_{2}, \ldots, \varphi_{A}\right\}=\frac{1}{\sqrt{A !}} \operatorname{det}\left[\varphi_{i}\left(\boldsymbol{r}_{j}\right)\right],
$$

where $\varphi_{i}$ is the single-particle wave packet which is a direct product of the deformed Gaussian spatial part [33], $\operatorname{spin}\left(\chi_{i}\right)$, and isospin $\left(\xi_{i}\right)$ parts,

$$
\begin{aligned}
\varphi_{i}(\boldsymbol{r}) & =\phi_{i}(\boldsymbol{r}) \otimes \chi_{i} \otimes \xi_{i}, \\
\phi_{i}(\boldsymbol{r}) & =\exp \left\{-\sum_{\sigma=x, y, z} v_{\sigma}\left(r_{\sigma}-\frac{Z_{i \sigma}}{\sqrt{\nu_{\sigma}}}\right)^{2}\right\}, \\
\chi_{i} & =a_{i} \chi_{\uparrow}+b_{i} \chi_{\downarrow}, \quad \xi_{i}=\text { proton or neutron. }
\end{aligned}
$$

The centroids of the Gaussian wave packets $\boldsymbol{Z}_{i}$, the direction of nucleon spin $a_{i}, b_{i}$, and the width parameter of the deformed Gaussian $v_{\sigma}$ are the variational parameters. The intrinsic wave function is projected to the eigenstate of the parity to investigate both of the positive- and negative-parity states,

$$
\Phi^{\pi}=P^{\pi} \Phi_{\text {int }}=\frac{1+\pi P_{x}}{2} \Phi_{\text {int }}, \quad \pi= \pm,
$$

where $P^{\pi}$ and $P_{x}$ denote parity projector and operator. Using this wave function, the variational energy is defined as

$$
E^{\pi}=\frac{\left\langle\Phi^{\pi}|H| \Phi^{\pi}\right\rangle}{\left\langle\Phi^{\pi} \mid \Phi^{\pi}\right\rangle}
$$

By the frictional cooling method [34], the variational parameters are determined so that $E^{\pi}$ is minimized. In this study, we add the constraint potential to the variational energy,

$$
E^{\prime \pi}=\frac{\left\langle\Phi^{\pi}|H| \Phi^{\pi}\right\rangle}{\left\langle\Phi^{\pi} \mid \Phi^{\pi}\right\rangle}+v_{\beta}\left(\langle\beta\rangle-\beta_{0}\right)^{2}+v_{\gamma}\left(\langle\gamma\rangle-\gamma_{0}\right)^{2},
$$

where $\langle\beta\rangle$ and $\langle\gamma\rangle$ are the quadrupole deformation parameters of the intrinsic wave function defined in Refs. [24,35], and $v_{\beta}$ and $v_{\beta}$ are chosen large enough that $\langle\beta\rangle$ and $\langle\gamma\rangle$ are equal to $\beta$ and $\gamma$ after the variation. By minimizing $E^{\prime \pi}$, we obtain the optimized wave function $\Phi^{\pi}(\beta, \gamma)=P^{\pi} \Phi_{\text {int }}(\beta, \gamma)$ which has the minimum energy for each set of $\beta$ and $\gamma$.
After the variational calculation, the eigenstate of the total angular momentum $J$ is projected out from $\Phi^{\pi}(\beta, \gamma)$,

$$
\begin{aligned}
\Phi_{M K}^{J^{\pi}}(\beta, \gamma) & =P_{M K}^{J} \Phi^{\pi}(\beta, \gamma) \\
& =\frac{2 J+1}{8 \pi^{2}} \int d \Omega D_{M K}^{J *}(\Omega) R(\Omega) \Phi^{\pi}(\beta, \gamma) .
\end{aligned}
$$

Here, $P_{M K}^{J}, D_{M K}^{J}(\Omega)$, and $R(\Omega)$ are the angular-momentum projector, the Wigner $D$ function, and the rotation operator, respectively. The integrals over Euler angles $\Omega$ are evaluated numerically.

Next, we perform the generator coordinate method (GCM) calculation by employing the quadrupole deformation parameters $\beta$ and $\gamma$ as the generator coordinate. The wave function of GCM reads

$$
\Psi_{M n}^{J^{\pi}}=\sum_{i} \sum_{K} c_{\mathrm{Kin}}^{J^{\pi}} \Phi_{M K}^{J^{\pi}}\left(\beta_{i}, \gamma_{i}\right),
$$

where the coefficients $c_{\text {Kin }}^{J^{\pi}}$ and eigenenergies $E_{n}^{J^{\pi}}$ are obtained by solving the Hill-Wheeler equation [36],

$$
\begin{aligned}
\sum_{i^{\prime} K^{\prime}} H_{K i K^{\prime} i^{\prime}}^{J^{\pi}} c_{K^{\prime} i^{\prime} n}^{J} & =E_{n}^{J^{\pi}} \sum_{i^{\prime} K^{\prime}} N_{K i K^{\prime} i^{\prime}}^{J^{\pi}} c_{K^{\prime} i^{\prime} n}^{J^{\pi}}, \\
H_{K i K^{\prime} i^{\prime}}^{J^{\pi}} & =\left\langle\Phi_{M K}^{J^{\pi}}\left(\beta_{i}, \gamma_{i}\right)|H| \Phi_{M K^{\prime}}^{J^{\pi}}\left(\beta_{i^{\prime}}, \gamma_{i^{\prime}}\right)\right\rangle, \\
N_{K i K^{\prime} i^{\prime}}^{J^{\pi}} & =\left\langle\Phi_{M K}^{J^{\pi}}\left(\beta_{i}, \gamma_{i}\right) \mid \Phi_{M K^{\prime}}^{J^{\pi}}\left(\beta_{i^{\prime}}, \gamma_{i^{\prime}}\right)\right\rangle .
\end{aligned}
$$

We also calculate the overlap between $\Psi_{M n}^{J^{\pi}}$ and the basis wave function of the GCM $\Phi_{M K}^{J^{\pi}}\left(\beta_{i}, \gamma_{i}\right)$,

$$
\left|\left\langle\Phi_{M K}^{J^{\pi}}(\beta, \gamma) \mid \Psi_{M n}^{J^{\pi}}\right\rangle\right|^{2} /\left\langle\Phi_{M K}^{J^{\pi}}(\beta, \gamma) \mid \Phi_{M K}^{J^{\pi}}(\beta, \gamma)\right\rangle,
$$

to discuss the dominant configuration in each state described by $\Psi_{M n}^{J^{\pi}}$.

\section{B. Single-particle orbits}

Nucleon single-particle energy and orbit are useful to investigate the motion of the valence neutrons around the core nucleus. For this purpose, we construct a single-particle Hamiltonian and calculate the neutron single-particle orbits in the intrinsic wave function $\Phi_{\text {int }}(\beta, \gamma)$. We first transform the single-particle wave packet $\varphi_{i}$ to the orthonormalized basis,

$$
\tilde{\varphi}_{\alpha}=\frac{1}{\sqrt{\lambda_{\alpha}}} \sum_{i=1}^{A} g_{i \alpha} \varphi_{i}
$$

Here, $\lambda_{\alpha}$ and $g_{i \alpha}$ are the eigenvalues and eigenvectors of the overlap matrix $B_{i j}=\left\langle\varphi_{i} \mid \varphi_{j}\right\rangle$. Using this basis, the singleparticle Hamiltonian is derived,

$$
\begin{aligned}
h_{\alpha \beta}= & \left\langle\widetilde{\varphi}_{\alpha}|t| \tilde{\varphi}_{\beta}\right\rangle+\sum_{\gamma=1}^{A}\left\langle\widetilde{\varphi}_{\alpha} \widetilde{\varphi}_{\gamma}\left|v^{N}+v^{C}\right| \widetilde{\varphi}_{\beta} \widetilde{\varphi}_{\gamma}-\widetilde{\varphi}_{\gamma} \widetilde{\varphi}_{\beta}\right\rangle, \\
& +\frac{1}{2} \sum_{\gamma, \delta=1}^{A}\left\langle\widetilde{\varphi}_{\gamma} \widetilde{\varphi}_{\delta}\left|\tilde{\varphi}_{\alpha}^{*} \widetilde{\varphi}_{\beta} \frac{\delta v^{N}}{\delta \rho}\right| \tilde{\varphi}_{\gamma} \widetilde{\varphi}_{\delta}-\tilde{\varphi}_{\delta} \tilde{\varphi}_{\gamma}\right\rangle .
\end{aligned}
$$

The eigenvalues $\epsilon_{s}$ and eigenvectors $f_{\alpha s}$ of $h_{\alpha \beta}$ give the single-particle energies and the single-particle orbits, $\widetilde{\phi}_{s}=\sum_{\alpha=1}^{A} f_{\alpha s} \widetilde{\varphi}_{\alpha}$. We also calculate the amount of the 
positive-parity component in the single-particle orbit,

$$
p^{+}=\left|\left\langle\widetilde{\phi}_{s}\left|\frac{1+P_{x}}{2}\right| \widetilde{\phi}_{s}\right\rangle\right|^{2},
$$

and angular momenta in the intrinsic frame,

$$
\begin{aligned}
& j(j+1)=\left\langle\widetilde{\phi}_{s}\left|j^{2}\right| \widetilde{\phi}_{s}\right\rangle, \quad\left|j_{z}\right|=\sqrt{\left\langle\widetilde{\phi}_{s}\left|j_{z}^{2}\right| \widetilde{\phi}_{s}\right\rangle}, \\
& l(l+1)=\left\langle\widetilde{\phi}_{s}\left|l^{2}\right| \widetilde{\phi}_{s}\right\rangle, \quad\left|l_{z}\right|=\sqrt{\left\langle\widetilde{\phi}_{s}\left|l_{z}^{2}\right| \widetilde{\phi}_{s}\right\rangle},
\end{aligned}
$$

which are used to discuss the properties of the single-particle orbits.

\section{C. $\alpha$ reduced width amplitude and decay width}

From the GCM wave function, we estimate the $\alpha$ reduced width amplitude (RWA) $y_{l j^{\pi^{\prime}}}(r)$, which is defined as

$$
y_{l j^{\pi}}(r)=\sqrt{\frac{A !}{4 !(A-4) !}}\left\langle\phi_{\alpha}\left[\phi_{\mathrm{Be}}\left(j^{\pi^{\prime}}\right) Y_{l 0}(\hat{r})\right]_{J^{\pi} M} \mid \Psi_{M n}^{J^{\pi}}\right\rangle,
$$

where $\phi_{\alpha}$ and $\phi_{\mathrm{Be}}\left(j^{\pi^{\prime}}\right)$ denote the wave functions for $\alpha$ particle and daughter nucleus ${ }^{10} \mathrm{Be}$ with spin-parity $j^{\pi^{\prime}}$. The square of the RWA $\left|r y_{l j \pi^{\prime}}(r)\right|^{2}$ is equal to the probability to observe the $\alpha$ particle and ${ }^{10} \mathrm{Be}$ with spin-parity $j^{\pi^{\prime}}$ at intercluster distance $r$ with relative orbital angular momentum $l$ in the ${ }^{14} \mathrm{C}$ described by the GCM wave function $\Psi_{M n}^{J^{\pi}}$.

Using RWA, the partial $\alpha$-decay widths for the decay process ${ }^{14} \mathrm{C}\left(J^{\pi}\right) \rightarrow \alpha+{ }^{10} \mathrm{Be}\left(j^{\pi^{\prime}}\right)$ is estimated as

$$
\Gamma_{l j^{\pi^{\prime}}}^{\alpha}=2 P_{l}(a) \gamma_{l j^{\pi^{\prime}}}^{2}(a), \quad P_{l}(a)=\frac{k a}{F_{l}^{2}(k a)+G_{l}^{2}(k a)},
$$

where $a$ denote the channel radius, and the penetration factor $P_{l}$ is given by the Coulomb regular and irregular wave functions $F_{l}$ and $G_{l}$. The wave number $k$ is given by the decay $Q$ value and the reduced mass as $k=\sqrt{2 \mu E_{Q}}$. The reduced width $\gamma_{l j^{\pi^{\prime}}}$ is

$$
\gamma_{l j^{\pi^{\prime}}}^{2}(a)=\frac{\hbar^{2}}{2 \mu a}\left[a y_{l j^{\pi^{\prime}}}(a)\right]^{2} .
$$

To calculate RWA with reduced computational cost, we employ the method given in Ref. [37], which suggests an approximation validated for sufficiently large intercluster distance $a$,

$$
\begin{aligned}
\left|a y_{l j^{\pi^{\prime}}}(a)\right|^{2} & \simeq \sqrt{\frac{\gamma}{2 \pi}}\left|\left\langle\Phi_{B B}^{J \pi}(a) \mid \Psi_{M n}^{J^{\pi}}\right\rangle\right|^{2}, \\
\gamma & =\frac{4(A-4)}{A} v_{B B},
\end{aligned}
$$

which means that RWA is reasonably approximated by the overlap between the GCM wave function and the Brink-Bloch wave function $\Phi_{B B}^{J^{\pi}}(a)$ composed of the ${ }^{10} \mathrm{Be}$ and $\alpha$ particle with the Gaussian width parameter $v_{B B}$. In this study, the channel radius $a$ is chosen as $5.2 \mathrm{fm}$, which is the same with that used for the $R$-matrix analysis of the experiment [29].

In the case of the present study, since ${ }^{10} \mathrm{Be}$ is deformed, it must be projected to the eigenstate of the angular momentum. Therefore, we constructed $\Phi_{B B}^{J^{\pi}}$ as follows: We first calculate the approximate intrinsic wave function $\psi_{\mathrm{Be}}$ for ${ }^{10} \mathrm{Be}$ by the
AMD energy variation in which the width parameter $v_{B B}$ is fixed to $0.16 \mathrm{fm}^{-2}$. We obtained two different configurations for ${ }^{10} \mathrm{Be}$; so-called $\pi^{2}$ and $\sigma^{2}$ configurations, which have valence neutrons in the $p$ shell and $s d$ shell, respectively. It is known that the former is dominant in the ground band and the latter is dominant in the excited band. Therefore, we regard the $\pi^{2}$ configuration as the ground band (the $0_{1}^{+}, 2_{1}^{+}$, and $4_{1}^{+}$states) configuration, while the $\sigma^{2}$ configuration as the excited band (the $0_{2}^{+}, 2_{3}^{+}$, and $4_{2}^{+}$states) configuration. Then, these wave functions are projected to the eigenstate of the angular momentum and combined with the $\alpha$ cluster wave function to constitute the intrinsic wave function of ${ }^{14} \mathrm{C}^{*}$ having ${ }^{10} \operatorname{Be}\left(0_{1}^{+}, 2_{1}^{+}, 4_{1}^{+}\right) \otimes \alpha$ and ${ }^{10} \operatorname{Be}\left(0_{2}^{+}, 2_{3}^{+}, 4_{2}^{+}\right) \otimes \alpha$ configurations,

$$
\left|\Phi_{B B}(a)\right\rangle=\left|\mathcal{A}\left\{\psi_{\alpha}\left(-\frac{10}{14} a\right) P_{m 0}^{j} \psi_{\mathrm{Be}}\left(\frac{4}{14} a\right)\right\}\right\rangle,
$$

where we assumed that $\psi_{\mathrm{Be}}$ is parity and axially symmetric, which is validated by the numerical check. Next, the reference wave function is constructed by the angular-momentum projection of the total system:

$$
\Phi_{B B}^{J \pi}(a)=\frac{1}{N} \frac{2 l+1}{2 J+1} \sum_{m} C_{l 0 j m}^{J m} P_{M m}^{J^{\pi}}\left|\Phi_{B B}(a)\right\rangle .
$$

Here $C_{l 0 j m}^{J m}$ and $N$ denotes the Clebsch-Gordan coefficient and the normalization factor, respectively. The summation over $m$ is needed to project the relative angular momentum between ${ }^{10} \mathrm{Be}$ and $\alpha$ particle to $l$.

Here we comment on the uncertainty of the reduced widths calculated by this method. There are two main sources of the uncertainty. The first is the choice of channel radius. In general, the calculated partial widths $\Gamma_{l j^{\pi}}^{\alpha}$ do not depend on the choice of the channel radius, if the GCM wave function has the correct asymptotic behavior. However, in the present calculation, we adopted the bound-state approximation in the GCM calculation and hence the wave function is not correct at large distance. In addition to this, we apply the approximate formula (20) by neglecting the antisymmetrization effect. Therefore, the approximation is not applicable for the small channel radius. We estimate it as $a<4 \mathrm{fm}$, which corresponds to the touching of the $\alpha$ and ${ }^{10} \mathrm{Be}$ clusters. As a result, the channel radius should be chosen at the moderate distance. We numerically checked the stability of the partial width $\Gamma_{l j^{\pi}}^{\alpha}$ and found that it is almost constant in the range of $a=5.0 \pm 1.0$ $\mathrm{fm}$, but it becomes smaller at larger distance and unstable at smaller distance.

Another source of the uncertainty is the ${ }^{10} \mathrm{Be}$ wave function. As already mentioned, the intrinsic wave function of ${ }^{10} \mathrm{Be}$ is approximated by a single Slater determinant with spherical Gaussian wave packets to reduce the computational cost. On the other hand, it has been shown that the property of ${ }^{10} \mathrm{Be}$ is reproduced by the "full calculation" [38,39] in which many Slater determinants are superposed (GCM) and the deformed Gaussian is used. Therefore, the validity of the approximation can be checked by comparing both calculations and calculating the overlap of the wave functions. By the full calculation, the binding energies of the ground and $0_{2}^{+}$states are calculated to be 65.8 and $60.1 \mathrm{MeV}$, and their radii are 2.55 and $3.12 \mathrm{fm}$, 
respectively. On the other hand, the present approximation gives the binding energies of 59.0 and $49.0 \mathrm{MeV}$ and the radii of 2.47 and $2.91 \mathrm{fm}$. The overlaps of the wave functions between the full and present calculations are 0.79 for the ground state and 0.43 for the $0_{2}^{+}$state. These results remark the importance of the use of the deformed Gaussian wave packet as emphasized in Ref. [33]. At the same time, we can say that the calculated reduced widths for the ${ }^{10} \mathrm{Be}\left(0_{1}^{+}, 2_{1}^{+}, 4_{1}^{+}\right) \otimes \alpha$ configurations should be reasonable and uncertainty is around $20 \%$ because the overlap of the ground state is large. On the other hand, the uncertainty for the ${ }^{10} \operatorname{Be}\left(0_{2}^{+}, 2_{3}^{+}, 4_{2}^{+}\right) \otimes \alpha$ configurations is as large as $50 \%$, which means the reduced width can be misestimated by a factor of two. However, we also note that this uncertainty does not qualitatively affect our discussion. As we see later, the positive-parity cluster states have much larger reduced widths than other states and, hence, the assignment of the positive-parity cluster bands are rather unique.

\section{RESULTS}

\section{A. Energy surface and intrinsic structures}

Figure 1(a) shows the energy surface as functions of quadrupole deformation parameters $\beta$ and $\gamma$ for $J^{\pi}=0^{+}$ states obtained by the constraint variational calculation and angular-momentum projection. The circles on the energy surfaces show the position of the energy minima.

The energy minimum of the $0^{+}$state is located at $(\beta, \gamma)=$ $\left(0.36,14^{\circ}\right)$ with the binding energy of $106.1 \mathrm{MeV}$. This intrinsic wave function is the most dominant component of the ground band as discussed in the next section. It is interesting that this minimum state is deformed, as seen in its intrinsic density distribution shown in Fig. 2(a), despite the neutron magic number $N=8$. However, the deformation is not large enough to break the neutron magicity because the last valence neutron occupies a $p$ wave, which can be deduced from the density distribution of the valence neutron orbit shown in Fig. 2(a).

In the oblate deformed region, the different structure that we call the triangular configuration appears. Fig. 2(b) shows the density distribution of the wave function located at $(\beta, \gamma)=\left(0.60,25^{\circ}\right)$. The proton density distribution has
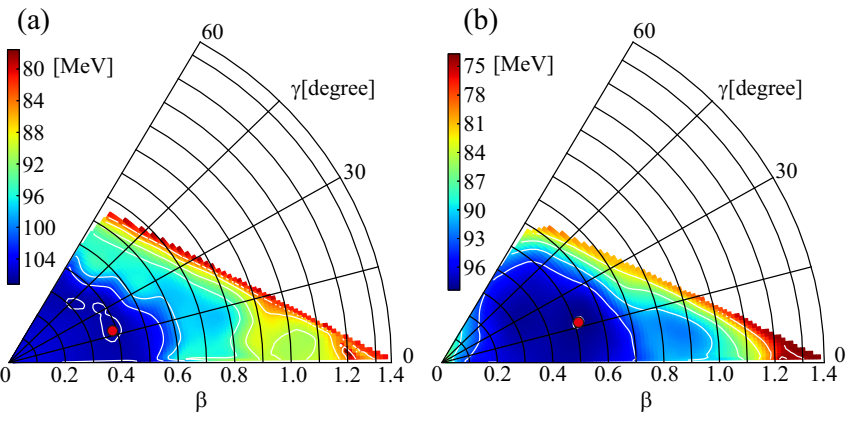

FIG. 1. The angular-momentum projected energy surface for (a) the $J^{\pi}=0^{+}$state and (b) $J^{\pi}=1^{-}$state as functions of quadrupole deformation parameters $\beta$ and $\gamma$. The circles show the position of the energy minima.

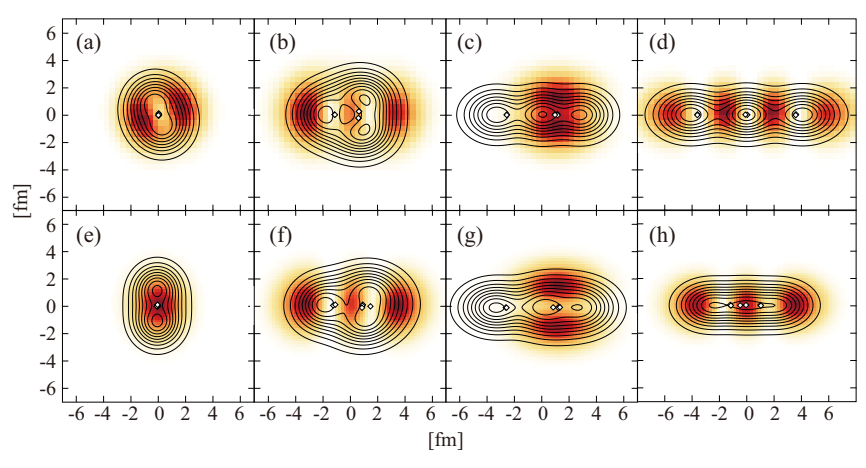

FIG. 2. The density distribution of (a)-(d) the positive states and (e)-(h) negative parity states. The contour lines show the proton density distributions. The color plots show the single-particle orbits occupied by the most weakly bound neutron. Open boxes show the centroids of the Gaussian wave packets describing protons.

a triangular shape showing the possible formation of a $3 \alpha$ cluster core with a triangle configuration. Indeed, as shown in the next section, this intrinsic wave function becomes the most dominant component of the triangular band, which has large $\alpha$ reduced widths. Owing to the parity asymmetric shape, the valence proton orbit is an admixture of the positive- and negative-parity component, as confirmed from the properties of the single-particle orbit listed in Table I. The table also shows that two valence neutrons occupy a positive-parity orbit ( $s d$ shell), indicating the $2 \hbar \omega$ excitation. Note that a similar configuration, i.e., a triangular $3 \alpha$ cluster core with $2 \hbar \omega$ excited valence neutrons, was also found in ${ }^{16} \mathrm{C}$ [27].

TABLE I. The properties of the most weakly bound proton and neutron orbits in the configurations shown in Figs. 2(a)-2(h). The column Occ. shows the number of the nucleon occupying the orbit. When two valence nucleons occupy the almost degenerate orbits, the single-particle properties are averaged and Occ. $=2$. Other columns show the single-particle energy $\varepsilon$ in $\mathrm{MeV}$, the amount of the positive-parity component $p^{+}$, and the angular momenta defined by Eqs. (14)-(16).

\begin{tabular}{|c|c|c|c|c|c|c|c|c|}
\hline & & Occ. & $\varepsilon$ & $p^{+}$ & $j$ & $\left|j_{z}\right|$ & $l$ & $\left|l_{z}\right|$ \\
\hline \multirow[t]{2}{*}{ (a) } & Proton & 2 & -17.4 & 0.00 & 1.5 & 1.5 & 1.1 & 1.0 \\
\hline & Neutron & 2 & -6.6 & 0.22 & 1.1 & 0.6 & 1.2 & 0.9 \\
\hline \multirow[t]{2}{*}{ (b) } & Proton & 2 & -14.1 & 0.08 & 1.6 & 1.5 & 1.2 & 1.0 \\
\hline & Neutron & 2 & -5.3 & 0.98 & 2.2 & 0.5 & 1.8 & 0.3 \\
\hline \multirow[t]{2}{*}{ (c) } & Proton & 2 & -12.5 & 0.97 & 2.2 & 0.5 & 2.0 & 0.2 \\
\hline & Neutron & 2 & -7.0 & 0.09 & 1.8 & 1.5 & 1.4 & 1.0 \\
\hline \multirow[t]{2}{*}{ (d) } & Proton & 2 & -15.6 & 0.99 & 2.5 & 0.5 & 2.3 & 0.1 \\
\hline & Neutron & 2 & -4.4 & 0.01 & 2.8 & 0.5 & 2.6 & 0.1 \\
\hline \multirow[t]{2}{*}{ (e) } & Proton & 2 & -16.0 & 0.00 & 1.5 & 1.4 & 1.1 & 1.0 \\
\hline & Neutron & 1 & -3.8 & 0.99 & 2.2 & 0.5 & 1.8 & 0.4 \\
\hline \multirow[t]{2}{*}{ (f) } & Proton & 1 & -12.6 & 0.53 & 1.9 & 0.9 & 1.6 & 0.8 \\
\hline & Neutron & 2 & -6.6 & 0.98 & 2.1 & 0.6 & 1.8 & 0.3 \\
\hline \multirow[t]{2}{*}{ (g) } & Proton & 1 & -12.4 & 0.72 & 2.3 & 0.9 & 2.1 & 0.6 \\
\hline & Neutron & 2 & -7.2 & 0.11 & 1.9 & 1.4 & 1.6 & 1.0 \\
\hline \multirow[t]{2}{*}{ (h) } & Proton & 1 & -13.1 & 0.52 & 1.9 & 1.0 & 1.6 & 0.8 \\
\hline & Neutron & 2 & -8.2 & 0.92 & 2.2 & 0.7 & 1.9 & 0.4 \\
\hline
\end{tabular}




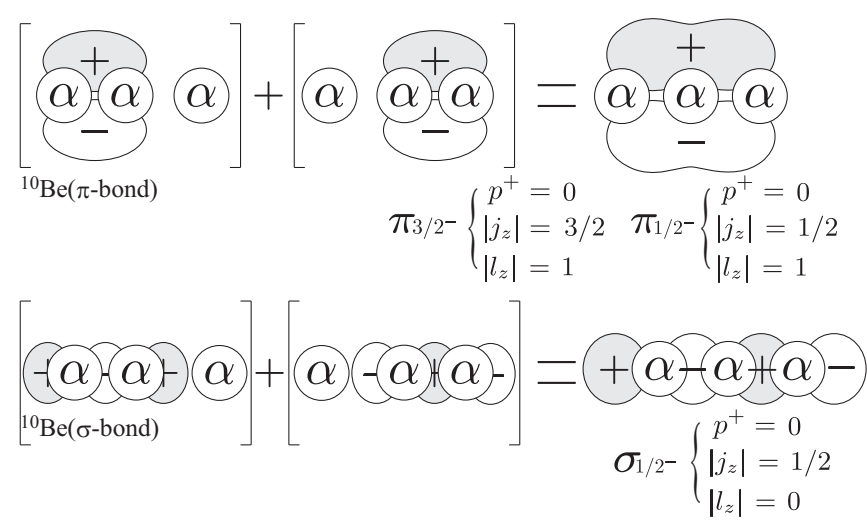

FIG. 3. The schematic figure showing the $\pi$ and $\sigma$ orbits around the linear chain. The combination of the $p$ orbits perpendicular to the symmetry axis generates $\pi$ orbits, while the combination of parallel orbits generates $\sigma$ orbits.

In the strongly deformed region, the linear-chain configurations appear. There is an energy plateau around the local energy minimum at $(\beta, \gamma)=(1.00,0)$ which approximately corresponds to the deformation ratio equal to $3: 1$. This intrinsic wave function is the most dominant component of the $\pi$-bond linear-chain band. As clearly seen in its density distribution shown in Fig. 2(c), this local energy minimum has pronounced $3 \alpha$ cluster structure with linear alignment. The properties of the proton single-particle orbit show that the last two protons are promoted into the $s d$ shell, which is because of the Pauli principle in the linear-chain configuration. The density distribution and properties of the valence neutron orbits show that they correspond to the $\pi$ orbit of the molecular-orbit picture, which is schematically illustrated in Fig. 3. Namely, the valence neutron orbit is a linear combination of the $p$ orbits perpendicular to the symmetry axis and has the angular momenta $\left|j_{z}\right|=1.5$ and $\left|l_{z}\right|=1.0$. We call this configuration the $\pi$-bond linear chain in the following. This property of the valence neutron orbit is common to that found in ${ }^{16} \mathrm{C}$ [27]. However, note that the $\pi$ orbit of ${ }^{14} \mathrm{C}$ does not have a paritysymmetric distribution but is localized between the center and right $\alpha$ clusters. In other words, this configuration is parity asymmetric and has a $\left({ }^{4} \mathrm{He}+{ }^{10} \mathrm{Be}\right)$-like structure, which is consistent with the discussion made in Refs. $[24,40]$. Because this linear-chain configuration and the triangular configuration explained above have asymmetric internal structures, we expect that the corresponding negative-parity partners may exist and constitute the inversion doublets.

With further increase of the deformation, the other linearchain configuration which we call a $\sigma$-bond linear chain appears around $(\beta, \gamma)=(1.27,0)$, which was not mentioned in Ref. [24]. This intrinsic wave function is the most dominant component of the $\sigma$-bond linear-chain band discussed in the next section. From the density distribution [Fig. 2(d)], it is clear that this configuration has another valence neutron orbit that corresponds to the $\sigma$ orbit, which is a linear combination of a $p$ orbit parallel to the symmetry axis and has the angular momenta $\left|j_{z}\right| \approx 0.50$ and $\left|l_{z}\right| \approx 0$. It is interesting to note that this configuration has a parity-symmetric shape and, hence, does not have its negative-parity partner.
The energy minimum of the energy surface for the $1^{-}$states [Fig. 1(b)] is located at $(\beta, \gamma)=\left(0.52,19^{\circ}\right)$ with the binding energy of $-98.2 \mathrm{MeV}$, and its density distribution is described in Fig. 2(e). From Table I, we see that this minimum has the same proton configuration with the positive-parity minimum, but a neutron is excited into the $s d$ shell from the $p$ shell $(1 p 1 h$ configuration).

In the deformed region, many different kinds of configurations appear. As reported in Ref. [24], we found that these configurations are strongly mixed to each other and that the unique band assignment of negative-parity states is impossible. Therefore, we do not discuss each configuration on the energy surface but explain the negative-parity partner of the cluster configurations found in the positive parity. Because the triangular configuration and the $\pi$-bond linear-chain of the positive parity are parity asymmetric, their counterparts appear in the negative parity. Figures 2(f) and $2(\mathrm{~g})$ show the triangular configuration and the $\pi$-bond linear-chain configuration in the negative-parity state located at $(\beta, \gamma)=\left(0.72,14^{\circ}\right)$ and $\left(1.05,3^{\circ}\right)$, respectively. Although the cluster cores are more distorted than the positive-parity states, their neutron singleparticle configurations are similar to their positive-parity counterparts. However, as already mentioned and explained in the next section, these cluster configurations do not form a single rotational band and are mixed with other configurations to yield several excited states. For example, the negative-parity $\pi$-bond linear-chain strongly mixes with the largely deformed noncluster states such as the configuration shown in Fig. 2(h).

\section{B. Excitation spectrum}

Figure 4 shows the spectrum of the positive-parity states obtained by the GCM calculation. The properties of the several selected states are listed in Table II. We classified the excited states which have larger $\alpha$ reduced widths than $0.08 \mathrm{MeV}^{1 / 2}$ as cluster states. The detail of the $\alpha$ reduced widths is given in the Sec. IV B. In the case of the positive-parity states, the cluster states are assigned in the rotational bands without uncertainty, because the band member states are connected by the strong E2 transitions as listed in Table III.

The ground state and the first-excited state $\left(2_{1}^{+}\right)$are dominantly composed of the configurations around the energy minimum of the energy surface. The ground state has the largest overlap with the configuration shown in Fig. 2(a), which amounts to 0.98 , and the calculated binding energy is $-106.3 \mathrm{MeV}$, which is reasonably close to the observed value of $-105.3 \mathrm{MeV}$. The excitation energy of the $2_{1}^{+}$state is also reasonably described. However, the calculated $B(E 2)$ strength overestimates the observed value, which may be due to the overestimation of the deformation. This overestimation of $B(E 2)$ is common to ${ }^{16} \mathrm{C}$ [27].

Owing to its triaxial deformed shape, the triangular configuration generates the rotational bands built on the $0_{2}^{+}$and $2_{3}^{+}$states that are shown by triangles in Fig. 4. We call them $K^{\pi}=0^{+}$and $2^{+}$bands, respectively, in the following, although the mixing of the $K$ quantum number in their GCM wave functions is not negligible. Compared to the linear-chain states, these bands have less-pronounced clustering and $\alpha$ clusters are considerably distorted; therefore, the band head 


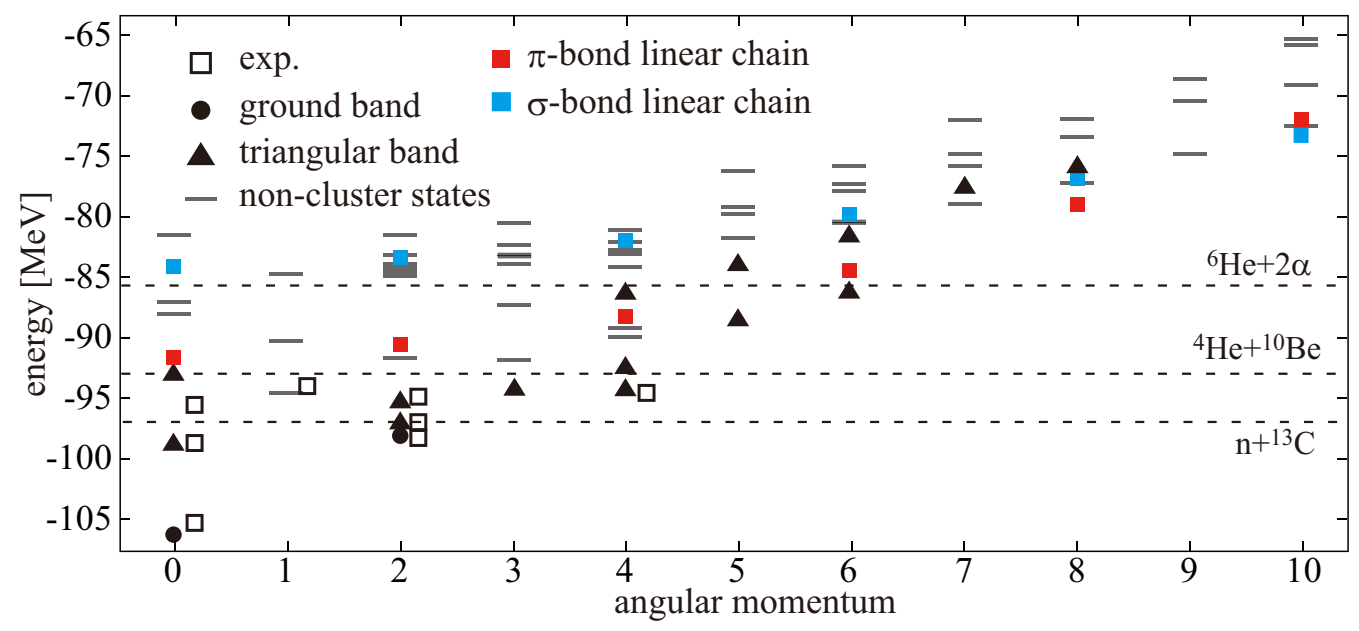

FIG. 4. The positive-parity energy levels up to $J^{\pi}=10^{+}$. Open boxes show the observed states with the definite spin-parity assignments taken from Ref. [41], and other symbols show the calculated result. The filled circles, triangles and filled boxes show the ground, triangular, and linear-chain bands, while lines show the noncluster states which have the reduced widths less than $0.08 \mathrm{MeV}^{1 / 2}$.

energies are well below the cluster thresholds. The member states have large overlap with the configuration shown in Fig. 2(b), which amounts to, for example, 0.91 in the case of the $0_{2}^{+}$state.

The linear-chain configurations generate two rotational bands in Fig. 4. The first one, which we call the $\pi$-bond linear-chain band, is built on the $0_{4}^{+}$state at $14.6 \mathrm{MeV}$ close to the $\alpha$-threshold energy and is composed of the $\pi$-bond linear-chain configurations. The band head state (the $0_{4}^{+}$state) has large overlap with the configuration shown in Fig. 2(c) which amounts to 0.87. The other band, which we call $\sigma$-bond linear-chain band, is built on the $0_{7}^{+}$state at 22.2 $\mathrm{MeV}$ (about 9.18 MeV above the $\alpha$ threshold) and is

TABLE II. Excitation energies $(\mathrm{MeV})$ and proton and neutron root-mean-square radii (fm) of several selected states. Numbers in the parentheses are the observed data $[41,42]$.

\begin{tabular}{lcccc}
\hline \hline Band & $J^{\pi}$ & $E_{x}$ & $r_{p}$ & $r_{n}$ \\
\hline Ground & $0_{1}^{+}$ & 0.00 & 2.53 & 2.58 \\
& $2_{1}^{+}$ & 8.41 & 2.58 & 2.69 \\
Triangular & & $(7.01)$ & $(2.34)$ & \\
$K^{\pi}=0^{+}$ & $0_{2}^{+}$ & 7.49 & 2.67 & 2.92 \\
& $2_{2}^{+}$ & 9.26 & 2.64 & 2.83 \\
Triangular & $4_{1}^{+}$ & 12.00 & 2.65 & 2.89 \\
$K^{\pi}=2^{+}$ & $2_{3}^{+}$ & 10.99 & 2.68 & 2.92 \\
& $3_{1}^{+}$ & 12.03 & 2.68 & 2.92 \\
$\pi$-bond & $4_{2}^{+}$ & 13.83 & 2.68 & 2.92 \\
Linear chain & $0_{4}^{+}$ & 14.64 & 3.27 & 3.20 \\
& $2_{5}^{+}$ & 15.73 & 3.37 & 3.28 \\
& $4_{5}^{+}$ & 17.98 & 3.33 & 3.24 \\
$\sigma$-bond & $6_{2}^{+}$ & 21.80 & 3.39 & 3.30 \\
Linear chain & $0_{7}^{+}$ & 22.16 & 3.91 & 4.12 \\
& $2_{10}^{+}$ & 22.93 & 4.02 & 4.21 \\
& $4_{11}^{+}$ & 24.30 & 3.97 & 4.15 \\
\hline \hline
\end{tabular}

composed of the $\sigma$-bond linear-chain configurations shown in Fig. 2(d). This intrinsic wave function has the largest overlap with the band-head state,which amounts to 0.99 . The $\pi$-bond linear-chain band is the candidate of the observed resonances and the comparison with the observation is discussed in the next section.

In the case of the negative-parity states shown in Fig. 5, it is found that the $\alpha$ cluster configurations are mixed with other configurations and yield many excited states having

TABLE III. The calculated in-band $B(E 2)$ strengths for the lowspin positive-parity states in units of $e^{2} \mathrm{fm}^{4}$. For the negative-parity states, the transitions between the low-spin cluster states (diamonds in Fig. 5) are shown and the transitions less than $10 e^{2} \mathrm{fm}^{4}$ are not shown. The number in parentheses is the observed data [43].

\begin{tabular}{lcc}
\hline \hline & $J_{i} \rightarrow J_{f}$ & $B\left(E 2 ; J_{i} \rightarrow J_{f}\right)$ \\
\hline Ground $\rightarrow$ ground & $2_{1}^{+} \rightarrow 0_{1}^{+}$ & $8.1(3.74)$ \\
Triangular $K^{\pi}=0^{+}$ & $2_{2}^{+} \rightarrow 0_{2}^{+}$ & 7.6 \\
$\rightarrow$ triangular $K^{\pi}=0^{+}$ & $4_{1}^{+} \rightarrow 2_{2}^{+}$ & 7.9 \\
& $6_{1}^{+} \rightarrow 4_{2}^{+}$ & 19.8 \\
Triangular $K^{\pi}=2^{+}$ & $3_{1}^{+} \rightarrow 2_{3}^{+}$ & 17.6 \\
$\rightarrow$ triangular $K^{\pi}=2^{+}$ & $4_{2}^{+} \rightarrow 3_{1}^{+}$ & 8.5 \\
& $4_{2}^{+} \rightarrow 2_{3}^{+}$ & 5.4 \\
$\pi$-bond linear chain & $2_{5}^{+} \rightarrow 0_{4}^{+}$ & 165.5 \\
$\rightarrow \pi$-bond linear chain & $4_{5}^{+} \rightarrow 2_{5}^{+}$ & 257.4 \\
& $6_{2}^{+} \rightarrow 4_{5}^{+}$ & 276.5 \\
$\sigma$-bond linear chain & $2_{10}^{+} \rightarrow 0_{7}^{+}$ & 441.9 \\
$\rightarrow \sigma$-bond linear chain & $4_{11}^{+} \rightarrow 2_{10}^{+}$ & 655.9 \\
Negative parity states & $3_{4}^{-} \rightarrow 1_{3}^{-}$ & 21.9 \\
& $3_{5}^{-} \rightarrow 1_{3}^{-}$ & 32.4 \\
& $3_{6}^{-} \rightarrow 1_{5}^{-}$ & 60.1 \\
& $3_{10}^{-} \rightarrow 1_{5}^{-}$ & 31.5 \\
& $5_{2}^{-} \rightarrow 3_{4}^{-}$ & 63.0 \\
& $5_{4}^{-} \rightarrow 3_{5}^{-}$ & 54.5 \\
& $5_{7}^{-} \rightarrow 3_{6}^{-}$ & 53.9 \\
\hline
\end{tabular}




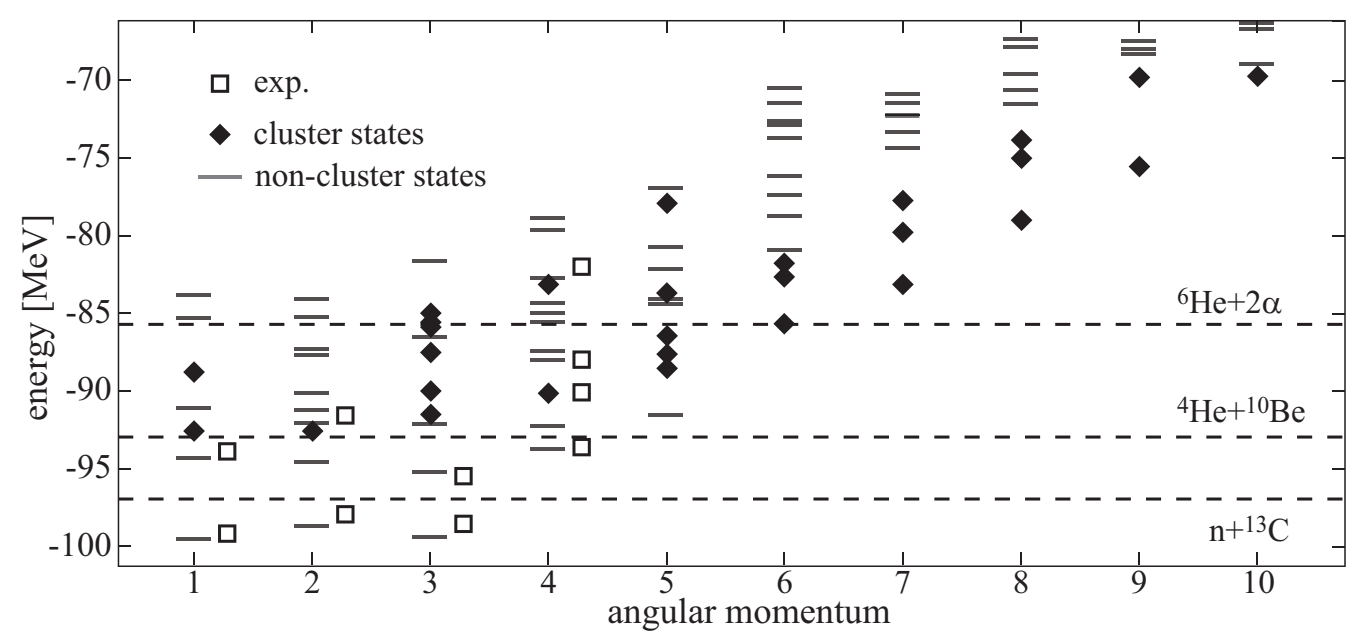

FIG. 5. The negative-parity energy levels up to $J^{\pi}=10^{-}$. Open boxes show the observed states with the definite spin-parity assignments taken from Ref. [41], and other symbols show the calculated result. The diamonds show the cluster states having non-negligible $\alpha$ reduced widths, while lines show the noncluster states which have the reduced widths less than $0.08 \mathrm{MeV}^{1 / 2}$.

non-negligible $\alpha$ reduced widths. As a result, the $E 2$ transition strengths are also spread to several states, and it makes the band assignment ambiguous. For example, as listed in Table III, there are two $3^{-}$states that strongly decay to the $1_{3}^{-}$ state. Therefore, applying the same standard with the positive parity, we classified the excited states which have larger $\alpha$ reduced widths than $0.10 \mathrm{MeV}^{1 / 2}$ as cluster states (diamond symbols). Those cluster states are shown by diamond symbols which are mainly composed of the configurations shown in Figs. 2(f)-2(h) and other noncluster configurations. For example, the $1_{3}^{-}$state has the largest overlap with the configuration shown in Fig. 2(h), which amounts to 0.93. But, at the same time, this state also has a large overlap with the triangular configuration shown in Fig. 2(f) and the $\pi$-bond linear-chain shown in Fig. 2(g), which amounts to 0.85 and 0.70 , respectively. This means that these excited states are the mixture of cluster states and noncluster states. The fragmentation of the cluster configurations can be more clearly seen in their $\alpha$ reduced widths, which are discussed in the next section.

\section{DISCUSSION}

\section{A. Excitation energies of linear-chain bands}

In this section, we focus on the excitation energies of the linear-chain bands and compare them with the experiments $[21,29,30]$. The results of the present calculation and the experimental candidates are summarized in Fig. 6. By the measurement of the ${ }^{9} \mathrm{Be}\left({ }^{7} \mathrm{Li}, d\right){ }^{14} \mathrm{C}$ reaction, von Oertzen et al. [21] reported a candidate of the linear-chain band whose band-head energy is below the $\alpha$-threshold energy. Freer et al. [29] and Fritsch et al. [30] independently measured ${ }^{4} \mathrm{He}+{ }^{10} \mathrm{Be}$ resonant scattering by using a radioactive ${ }^{10} \mathrm{Be}$ beam and reported the candidates above the threshold energy. The resonance energies of the $4^{+}$state reported by Freer et al. and Fritsch et al. are close to each other, but those of the $2^{+}$state differ. However, it must be kept in mind that the assignment of the $2^{+}$state by Freer et al. is tentative, as mentioned in their report.
Then, we see that the calculated energy of the $\pi$-bond linear chain is close to the resonances observed in the ${ }^{4} \mathrm{He}+{ }^{10} \mathrm{Be}$ resonant scattering except for the tentatively assigned $2^{+}$state. In addition, as discussed in the next section, the $\alpha$ reduced widths of the $\pi$-bond linear chain and those observed resonances are close to each other. Hence, we conclude that the resonances observed in the ${ }^{4} \mathrm{He}+{ }^{10} \mathrm{Be}$ resonant scattering should be the $\pi$-bond linear chain. The excited states reported by von Oertzen et al. are approximately $5 \mathrm{MeV}$ lower than the $\pi$-bond linear chain, and it energetically corresponds to the triangular band. The measurement of the $\alpha$ widths of those candidate will make this assignment sure. The $\sigma$-bond linear chain is energetically located higher than any observed resonances and does not have the experimental counterpart. As we see later, the $\sigma$-bond linear chain is dominantly composed of the ${ }^{4} \mathrm{He}+{ }^{10} \mathrm{Be}\left(0_{2}^{+}\right)$and ${ }^{4} \mathrm{He}+{ }^{10} \mathrm{Be}\left(2_{3}^{+}\right)$component. Therefore, we consider that it is not easy to populate this band by ordinary transfer reaction or resonant scattering.

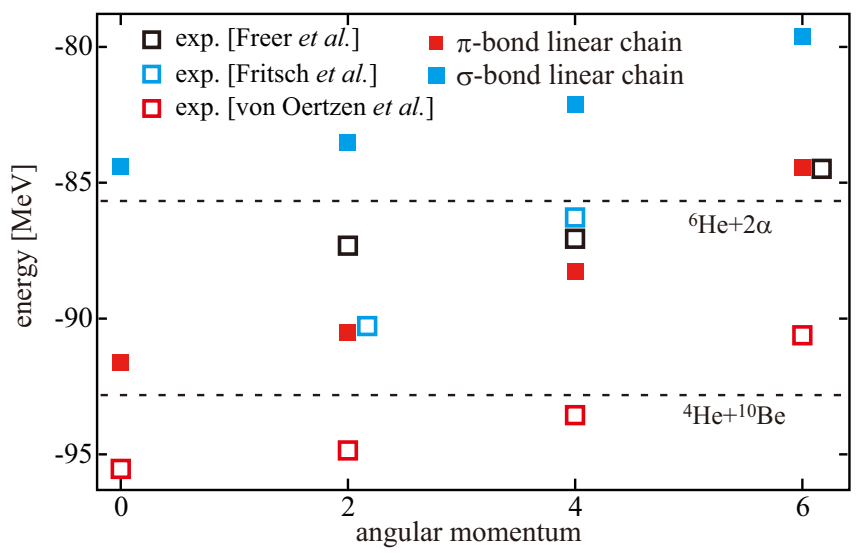

FIG. 6. The calculated and observed linear-chain candidates in positive parity. Open boxes show the observed data reported by Refs. $[21,29,30]$. Filled boxes show the energies of the $\pi$-bond and $\sigma$-bond linear-chain states. 


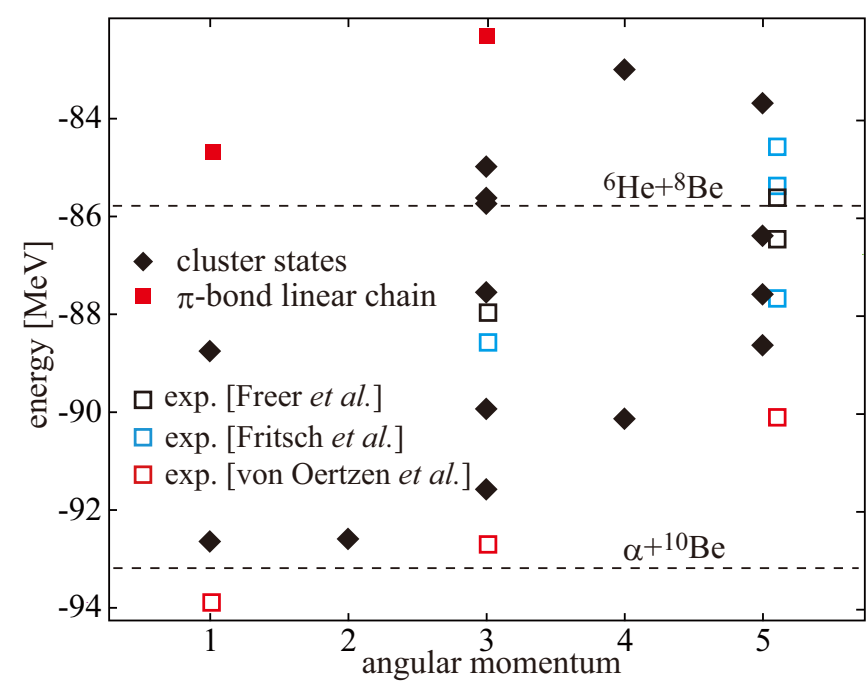

FIG. 7. The calculated cluster states and observed linear-chain candidates in negative parity. Open boxes show the observed data reported by Refs. [21,29,30]. Filled diamonds are the excited states with cluster configuration whose $\alpha$ reduced widths are larger than $0.08 \mathrm{MeV}^{1 / 2}$. Red boxes shows the $\pi$-bond linear-chain projected to negative parity.

Figure 7 summarizes the negative-parity results. In contrast to the positive parity, there are so many excited states with non-negligible reduced widths in the theoretical result. As a result, the correspondence between the theory and experiment is not unique. We also performed an additional test calculation. We pickup the $\pi$-bond linear-chain configuration with positive parity shown in Fig. 2(c) and artificially project it to the negative parity to estimate the energy of the ideal $\pi$-bond linear chain with negative parity. The results is shown by the red filled boxes in Fig. 7. We see that the energy of the ideal linear chain is too high to be assigned to the observed resonances. Thus, the present calculation does not support the formation of the linear chain in negative parity.

\section{B. Reduced widths}

Figure 8 shows the $\alpha$ reduced widths of several selected low-spin states with positive parity. The decay channels are indicated as $\left[{ }^{10} \mathrm{Be}\left(j^{\pi}\right) \otimes l\right]$ where $j^{\pi}$ and $l$ denote the angular momenta of the ${ }^{10} \mathrm{Be}$ and the relative motion between ${ }^{10} \mathrm{Be}$ and $\alpha$ particle, respectively. Here, except for Fig. $8(\mathrm{e}),{ }^{10} \mathrm{Be}$ is assumed to have two neutrons in a $\pi$ orbit, i.e., the ground band [44].

There are two prominent features to be noted in these results: The first is the magnitude of the reduced widths. The $\pi$-bond linear-chain band (the $0_{4}^{+}, 2_{5}^{+}, 4_{5}^{+}$, and $6_{2}^{+}$states) have large reduced widths compared to the triangular bands and the ground state. It is also noted that the $\alpha$ reduced widths of other excited states are also smaller than the $\pi$-bond linear-chain band, and even smaller than or comparable to the triangular bands. Hence, in the calculated energy region, the $\pi$-bond linear chain band has the largest reduced width. In Figs. 8(b)-8(d), the observed reduced widths of the linearchain candidates [29] are also shown for $2^{+}, 4^{+}$, and $6^{+}$states.
Since the decay to the ${ }^{10} \mathrm{Be}$ ground state was assumed in the $R$-matrix analysis made in Ref. [29], those values may be compared with the calculated results for the $\left[{ }^{10} \mathrm{Be}\left(0_{1}^{+}\right) \otimes l\right]$ channel, and we see that only the $\pi$-bond linear-chain band can explain the magnitude of the observed reduced widths. Thus, both of the observed excitation energies and reduced widths are reasonably explained by the $\pi$-bond linear-chain band, and we consider that the linear-chain formation in the positive-parity looks plausible.

It is also interesting to note that the other linear-chain band, i.e., the $\sigma$-bond linear-chain band, has suppressed reduced widths despite its prominent $\alpha$ clustering. The reason is simple. Because the $\sigma$-bond linear-chain band does not have valence neutron in a $\pi$ orbit, it is orthogonal to the decay channels to the ${ }^{10} \mathrm{Be}$ ground state that has a $\pi$-orbit neutron. This is confirmed in Fig. 8(e) where the reduced widths for the decays to the ${ }^{10} \mathrm{Be}$ with $\sigma$ bond [the excited rotational band, $\left.{ }^{10} \operatorname{Be}\left(0_{2}^{+}, 2_{3}^{+}, 4_{2}^{+}\right)\right]$are shown. Since other bands do not have valence neutrons in a $\sigma$ orbit, their reduced widths are suppressed, and only the $\sigma$-bond linear-chain band has large widths. Very interestingly, an experiment [45] reported the possible existence of the resonances around $E_{x}=22$ and 24 $\mathrm{MeV}$, which primary decays to ${ }^{10} \mathrm{Be}\left(0_{2}^{+}\right)$. Since the reported energies and the decay pattern are in good accordance with the $\sigma$-bond linear-chain, further investigation is very fascinating and in need.

Another point to be noted is the decay pattern of the $\pi$-bond linear-chain band. The reduced widths in the $\left[{ }^{10} \mathrm{Be}\left(2_{1}^{+}\right) \otimes l\right]$ channels are as large as or even larger than those in the $\left[{ }^{10} \mathrm{Be}\left(0_{1}^{+}\right) \otimes l\right]$ channel. This dominance of the ${ }^{10} \mathrm{Be}\left(2_{1}^{+}\right)$ component in the $\pi$-bond linear-chain band is due to its unique structure. When three $\alpha$ particles are linearly aligned, because of the strong angular correlation between $\alpha$ particles, the ${ }^{10} \mathrm{Be}\left(2_{1}^{+}\right)$and ${ }^{10} \mathrm{Be}\left(4_{1}^{+}\right)$components become large. This property is in contrast with the Hoyle state where $\alpha$ particles are mutually orbiting with $l=0$ and. hence, the ${ }^{8} \mathrm{Be}\left(0_{1}^{+}\right)$component dominates [8]. Similar properties of the linear-chain configuration were also discussed for ${ }^{12} \mathrm{C}[46]$. Therefore, if the large contamination of the ${ }^{10} \mathrm{Be}\left(2_{1}^{+}\right)$component is confirmed, it will be strong evidence for the linear-chain formation.

The ${ }^{10} \mathrm{Be}\left(2_{1}^{+}\right)$component in the triangular bands also shows an interesting feature. There are two triangular bands with $K^{\pi}=0^{+}$and $K^{\pi}=2^{+}$. The $0_{2}^{+}, 2_{2}^{+}$, and $4_{1}^{+}$states are the member of the $K^{\pi}=0^{+}$band, while the $2_{3}^{+}, 4_{2}^{+}$and $6_{1}^{+}$ are member of the $K^{\pi}=2^{+}$band. Here, we clearly see that the ${ }^{10} \mathrm{Be}\left(0_{1}^{+}\right)$component is dominant in the $K^{\pi}=0^{+}$band, while the ${ }^{10} \mathrm{Be}\left(2_{1}^{+}\right)$component is dominant in the $K^{\pi}=2^{+}$ band. This feature is explained by Fig. 9. In the triangular bands, ${ }^{4} \mathrm{He}$ and ${ }^{10} \mathrm{Be}$ are placed in a triangular shape and the intrinsic $z$ axis is chosen to be perpendicular to the deformation axis of ${ }^{10} \mathrm{Be}$. Since the $K$ quantum number is the angular momentum directed to the intrinsic $z$ axis, $K$ must be equal to the angular momentum of ${ }^{10} \mathrm{Be}$. This makes the difference in the amount of the ${ }^{10} \mathrm{Be}\left(2_{1}^{+}\right)$component in the $K^{\pi}=0^{+}$and $2^{+}$bands.

For negative parity, we show the states that have reduced widths larger than $0.08 \mathrm{MeV}^{1 / 2}$ in Figs. 8(f)-8(h). We can see that there are many states which have non-negligible $\alpha$ 

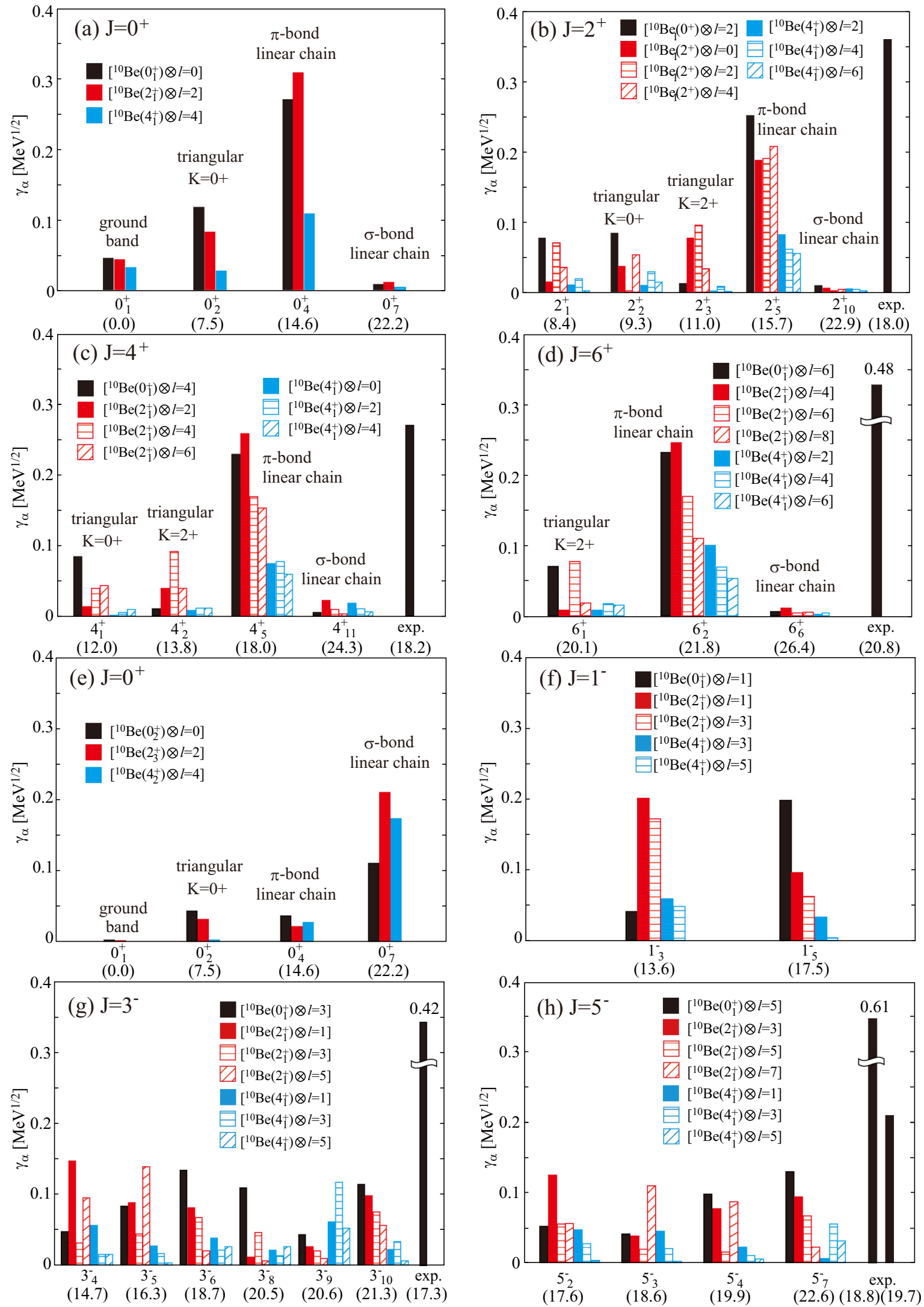

FIG. 8. The calculated $\alpha$-decay reduced widths compared with the observed widths reported in Ref. [29]. Panels (a)-(d) show the decay of the positive-parity states to the ground band of ${ }^{10} \mathrm{Be}\left(\pi\right.$-bonded $\left.{ }^{10} \mathrm{Be}\right)$. Panel (e) shows the decay of the $0^{+}$states to the excited band of ${ }^{10} \mathrm{Be}\left(\sigma\right.$-bonded $\left.{ }^{10} \mathrm{Be}\right)$. Panels (f)-(h) show the decay of the negative-parity states to the ground band of ${ }^{10} \mathrm{Be}\left(\pi\right.$-bonded $\left.{ }^{10} \mathrm{Be}\right) . \mathrm{Numbers}$ in parentheses show the excitation energy. The channel radius $a$ is $5.2 \mathrm{fm}$. 


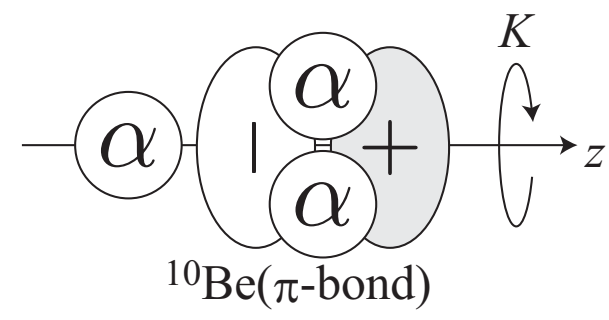

FIG. 9. The schematic figure explains the relationship between the $K$ quantum number and the angular momentum of ${ }^{10} \mathrm{Be}$.

reduced widths and are not able to identify the linear-chain band. As already mentioned, the linear-chain configurations are mixed with the noncluster configurations and yield many excited states, as found in Ref. [24]. We also see that none of the calculated state can explain the observed reduced widths that are twice as large as the present results. This requires further theoretical study of the negative-parity states, although the current result looks negative to the linear-chain formation in the negative-parity.

\section{SUMMARY}

To investigate the existence of the linear-chain state, we have studied the excited states of ${ }^{14} \mathrm{C}$ based on the AMD calculations.

In the positive-parity states, we found that three different configurations appear depending on the magnitude of the deformation and the valence neutron configurations. At the oblate deformed region, the triangular configuration of $3 \alpha$ cluster was obtained, while at strong deformed prolate region, two different linear-chain configurations with valence neutrons in the $\pi$ orbit and $\sigma$ orbit were obtained.

These cluster configurations generate clear rotational bands. The $\pi$-bond linear chain generates a rotational band around the $\alpha$-threshold energy, while triangular and $\sigma$-bond linear chain generates rotational bands well below and well above the threshold. The energy of the $\pi$-bond linear chain is in reasonable agreement with the resonances observed by the ${ }^{4} \mathrm{He}+{ }^{10} \mathrm{Be}$, while the triangular band is close to the excited states observed by the ${ }^{9} \mathrm{Be}\left({ }^{7} \mathrm{Li}, d\right){ }^{14} \mathrm{C}$ reaction. The analysis of the $\alpha$ reduced width confirms the assignment of the $\pi$-bond linear chain to the observed resonances, because the calculated and measured widths showed reasonable agreement. Thus, the positive-parity linear-chain formation in ${ }^{14} \mathrm{C}$ looks plausible. Furthermore, the calculation predicts that the $\pi$-bond linearchain will also decay to the ${ }^{10} \mathrm{Be}\left(2_{1}^{+}\right)$as well as to the ${ }^{10} \mathrm{Be}\left(0_{1}^{+}\right)$. This characteristic decay pattern will be, if measured, more evidence of the linear-chain formation.

In the negative-parity states, the negative-parity partners of the cluster states were also obtained by the energy variation. However, because of the mixing with the noncluster configurations, these cluster configurations do not correspond to a single eigenstate in excited states. As a result, many excited states that have sizable $\alpha$ reduced width are obtained, and it makes the correspondence between the theory and experiment ambiguous. Thus, the present result is negative for the linear-chain formation in negative parity, although further studies are in need to identify the structure of the observed negative-parity resonances.

\section{ACKNOWLEDGMENTS}

The authors acknowledges the fruitful discussions with Dr. Suhara, Dr. Kanada-En'yo, Dr. Fritsch, Mr. Koyama, Dr. H. Otsu, Dr. Li, and Dr. Ye. One of the authors (M.K.) acknowledges the support by the Grants-in-Aid for Scientific Research on Innovative Areas from MEXT (Grant No. 2404:24105008) and JSPS KAKENHI Grant No. 16K05339. The other author (T.B.) acknowledges the support by JSPS KAKENHI Grant No. 16J04889.
[1] E. Uegaki, S. Okabe, Y. Abe, and H. Tanaka, Prog. Theor. Phys. 57, 1262 (1977); 62, 1621 (1979).

[2] M. Kamimura, Nucl. Phys. A 351, 456 (1981).

[3] P. Descouvemont and D. Baye, Phys. Rev. C 36, 54 (1987).

[4] Y. Kanada-En'yo, Phys. Rev. Lett. 81, 5291 (1998).

[5] A. Tohsaki, H. Horiuchi, P. Schuck, and G. Röpke, Phys. Rev. Lett. 87, 192501 (2001).

[6] Y. Funaki, A. Tohsaki, H. Horiuchi, P. Schuck, and G. Röpke, Phys. Rev. C 67, 051306 (2003).

[7] M. Chernykh, H. Feldmeier, T. Neff, P. von Neumann-Cosel, and A. Richter, Phys. Rev. Lett. 98, 032501 (2007).

[8] Y. Funaki, H. Horiuchi, and A. Tohsaki, Prog. Part. Nucl. Phys. 82, 78 (2015).

[9] H. Morinaga, Phys. Rev. 101, 254 (1956).

[10] M. Seya, M. Kohno, and S. Nagata, Prog. Theor. Phys. 65, 204 (1981).

[11] W. von Oertzen, Z. Phys. A 354, 37 (1996); 357, 355 (1997).

[12] N. Itagaki and S. Okabe, Phys. Rev. C 61, 044306 (2000).

[13] Y. Kanada-En'yo, H. Horiuchi, and A. Doté, Phys. Rev. C 60, 064304 (1999).
[14] Y. Kanada-En'yo, M. Kimura, and H. Horiuchi, C. R. Phys. 4, 497 (2003).

[15] Y. Kanada-En'yo, M. Kimura, and A. Ono, Prog. Theor. Exp. Phys. 2012, 01 A202 (2012).

[16] N. Itagaki, S. Okabe, K. Ikeda, and I. Tanihata, Phys. Rev. C 64, 014301 (2001).

[17] B. J. Greenhalgh et al., Phys. Rev. C 66, 027302 (2002).

[18] W. von Oertzen and H. G. Bohlen, C. R. Phys. 4, 465 (2003).

[19] H. G. Bohlen et al., Phys. Rev. C 68, 054606 (2003).

[20] N. I. Ashwood et al., Phys. Rev. C 70, 064607 (2004).

[21] W. von Oertzen et al., Eur. Phys. J. A 21, 193 (2004).

[22] N. Itagaki, W. von Oertzen, and S. Okabe, Phys. Rev. C 74, 067304 (2006).

[23] D. L. Price et al., Phys. Rev. C 75, 014305 (2007).

[24] T. Suhara and Y. Kanada-En'yo, Phys. Rev. C 82, 044301 (2010).

[25] J. Maruhn, N. Loebl, N. Itagaki, and M. Kimura, Nucl. Phys. A 833, 1 (2010).

[26] N. Furutachi and M. Kimura, Phys. Rev. C 83, 021303(R) (2011).

[27] T. Baba, Y. Chiba, and M. Kimura, Phys. Rev. C 90, 064319 (2014). 
[28] P. W. Zhao, N. Itagaki, and J. Meng, Phys. Rev. Lett. 115, 022501 (2015).

[29] M. Freer et al., Phys. Rev. C 90, 054324 (2014).

[30] A. Fritsch et al., Phys. Rev. C 93, 014321 (2016).

[31] A. Volkov, Nucl. Phys. 74, 33 (1965).

[32] J. F. Berger, M. Girod, and D. Gogny, Comput. Phys. Commun. 63, 365 (1991).

[33] M. Kimura, Phys. Rev. C 69, 044319 (2004).

[34] Y. Kanada-En'yo and H. Horiuchi, Prog. Theor. Phys. 93, 115 (1995).

[35] M. Kimura, R. Yoshida, and M. Isaka, Prog. Theor. Phys. 127, 287 (2012).

[36] D. L. Hill and J. A. Wheeler, Phys. Rev. 89, 1102 (1953).

[37] Y. Kanada-En'yo, T. Suhara, and Y. Taniguchi, Prog. Theor. Exp. Phys. 2014, 73D02 (2014).
[38] H. Homma, M. Isaka, and M. Kimura, Phys. Rev. C 91, 014314 (2015).

[39] M. Isaka and M. Kimura, Phys. Rev. C 92, 044326 (2015).

[40] T. Suhara and Y. Kanada-En'yo, Phys. Rev. C 84, 024328 (2011).

[41] F. Ajzenberg-Selove, Nucl. Phys. A 523, 1 (1991).

[42] I. Angelia and K. P. Marinovab, At. Data Nucl. Data Tables 99, 69 (2013).

[43] S. Raman, C. W. Nestor Jr., and P. Tikkanen, At. Data Nucl. Data Tables 78, 1 (2001).

[44] D. L. Powell, G. M. Crawley, B. V. N. Rao, and B. A. Robson, Nucl. Phys. A 147, 65 (1970).

[45] Z. Y. Tian et al., arXiv:1607.00157 [Chinese Phys. C. (to be published)].

[46] Y. Suzuki, H. Horiuchi, and K. Ikeda, Prog. Theor. Phys. 47, 1517 (1972). 